\title{
Protocols and resource allocation for the two- way relay channel with half-duplex terminals
}

\author{
Adrian Agustin, Member IEEE, Josep Vidal, Member IEEE and Olga Muñoz
}

\begin{abstract}
The two-way relay channel (TWRC) describes the communication between two terminals sharing a common relay. In this work we compare different protocols for the TWRC with a half-duplex relay (forwarding and protocol I) and transmission schemes (Network Coding Relaying with random binning and XOR precoding), when the relay works in decode-and-forward and without channel state information at the transmitters. We provide the optimal resource allocation (in terms of phase duration, data rate and power allocation) when there is an individual or sum-average power constraint over the terminals. Closed-form solutions are derived whenever it is possible, while for others cases we show that the resource allocation can be formulated as a convex problem, which means that the solution can be obtained by low-complexity interior point methods.
\end{abstract}

Index Terms - Cooperative transmissions, relay-assisted transmission, two-way relay channel

\section{INTRODUCTION}

Relay-assisted transmission has been a rapidly evolving research topic in recent years thanks to its distinct capability to provide robustness in the wireless communications. In a system with a terminal (source) that wants to transmit information to another terminal (destination), an additional terminal, the relay, comes up for helping in the transmission. This channel named one-way relay channel (OWRC) because the communication is unidirectional, was first introduced in [1] and some capacity expressions are given in [2]. The benefits of this type of transmission in wireless channels in terms of diversity gain are presented in [3][4].

The typical transmission modes at relay can be amplify-andforward (AF), decode-and-forward (DF) and compress-andforward (CF). Moreover, the relay must work under halfduplex mode due to the current state of the radio technology. Therefore, the relay-assisted transmission must be divided in orthogonal phases where the relay is receiving, denoted by relay-receive phase or phase $I$, or the relay is transmitting, referenced by relay-transmit phase or phase II. The transmission is carried out under various (up to four) protocols depending on which terminals are active in each phase, see for example the definition used in [5] [6].

This work was supported in part by the European Union through project ROCKET ICT-2007-1-215282 and FEDER funds, and by the Spanish/Catalan Science and Technology Commissions through projects: 2005SGR-00639, TEC2006-06481/TCM, TEC2004-04526 and CONSOLIDER CSD200800010 COMONSENS

A.Agustin, J.Vidal and O.Muñoz are with the Signal Theory and Comm. Dept. at UPC,Spain. Email \{adrian.agustin, josep.vidal, olga.munoz\}@upc.edu
In order to enhance the spectral efficiency of the halfduplex relay communications the two-way relay channel (TWRC) has been introduced in [7],[8] and [9]. In this type of channel, also named bidirectional relaying, two terminals exchange simultaneously their messages through the same half-duplex relay. Notice that both terminals work as sources and destinations, but not simultaneously.

In contrast to the protocols defined for OWRC, [5],[6], only two protocols are devised for the TWRC, forwarding and protocol $I$ (in the sense of the one defined in [4]). Other protocols like those defined in [14] require the simultaneous transmission from terminals and relay, an impossible scenario with half-duplex terminals.
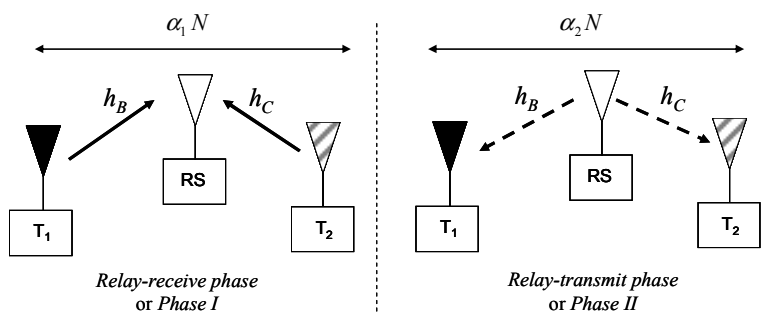

Figure 1. Forwarding protocol for the TWRC. Terminals $\mathrm{T}_{1}$ and

$\mathrm{T}_{2}$ exchange messages and the relay (RS) help both terminals.

The forwarding protocol is sketched in Figure 1 see for example [8],[10],[11] (two-step scheme). The transmission consists of two phases. In the first phase both terminals $\left(T_{1}\right.$ and $\mathrm{T}_{2}$ ) transmit simultaneously to the relay (RS), while in the second phase the relay transmits to both terminals. This protocol is named forwarding because the direct link between terminals $\mathrm{T}_{1}$ and $\mathrm{T}_{2}$ is not used.

On the other hand, protocol I presented in Figure 2 allows terminal $T_{1}\left(T_{2}\right)$ to receive a signal directly from $T_{2}\left(T_{1}\right)$ and also from the relay. This is done at the cost of designing the communication in three orthogonal phases, two phases $I$ and one phase II. Now, terminal $\mathrm{T}_{1}$ transmits to the relay and terminal $\mathrm{T}_{2}$ in the first phase (phase $I$ ). Afterwards, terminal $\mathrm{T}_{2}$ transmits to terminal $\mathrm{T}_{1}$ and the relay in the second phase (phase I). Finally, the relay (RS) transmits to both terminals (phase II). This protocol has been considered in [7] and [11] (three-step scheme).

It is important to realize that under protocol $I$ the message is transmitted through different links, hence, in principle some diversity gain is expected compared with the forwarding protocol. However, due to the orthogonal duplexing, protocol $I$ requires more orthogonal transmission phases. Therefore, it is difficult to anticipate which protocol is the best in terms of bits/channel use. 


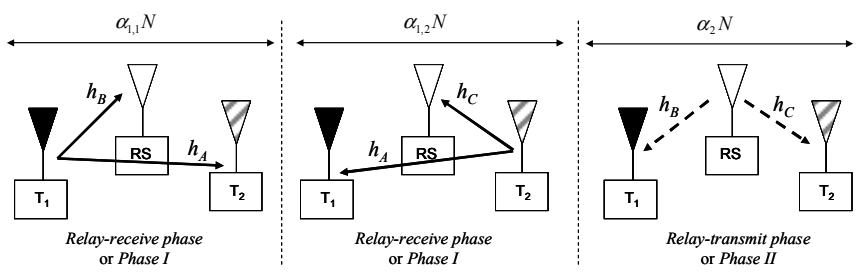

Figure 2. Protocol I for the TWRC.

One of the transmission strategies at the relay for the TWRC is based on the network coding relaying (NCR), where the relay generates a common signal intended to both terminals from the messages received in the first phase employing the random binning technique. The achievable rate region is shown in [10][12][13]. Similarly, there is a simple technique based on bit-wise XOR operation [7][11] that gets significant results although not as good as the NCR. The aforementioned references used to work with static resource allocation where all transmission phases present the same duration (except for [10],[11] with XOR precoding) and terminals present individual power constraints. This work concentrates on elucidating which protocol (forwarding or protocol $I$ ) gets the best performance based on individual power constraint (max-power) or a sum-average power constraint. The latter case allows us analyzing the efficiency of each transmission scheme. We investigate the optimal resource allocation in terms of duration of phases, transmitted power and data rate. The main contributions of the paper are:

- We provide closed-form expressions for the optimal resource allocation whenever is possible. Otherwise, we formulate the resource allocation as a convex problem [15], hence the solution is unique.

- We show how far the results obtained with NCR are from the ones using XOR precoding.

- We compare both protocols with the best protocol of the one-way relay channel (OWRC) [14] for a given scenario.

\section{SCENARIO AND Signal Model}

The transmission is carried out in frames of length $T_{\text {frame }}$ with $N$ channel uses and normalized bandwidth equal to $1 \mathrm{~Hz}$. For the forwarding protocol (see Figure 1) there are two phases where both terminals are simultaneously transmitting or receiving, the duration of each phase is proportional to $\alpha_{1}$ and $\alpha_{2}$, respectively. For protocol $I$, three phases are defined (see Figure 2). The first two are proportional to $\alpha_{1,1}$ and $\alpha_{1,2}$ (those correspond to the phase $I$ for each terminal) and the last phase is proportional to $\alpha_{2}$ (phase II).

Terminals $T_{1}, T_{2}$ and $R S$ are equipped with a single antenna. The channel gains of the direct $\left(\mathrm{T}_{1}-\mathrm{T}_{2}\right), \mathrm{T}_{1}-\mathrm{RS}$ and $\mathrm{RS}-\mathrm{T}_{2}$ links are referenced by the variables $h_{\mathrm{A}}, h_{\mathrm{B}}$ and $h_{\mathrm{C}}$ in Figure 1 and Figure 2 and remain constant over the whole transmission. In general, the perfect channel state information (CSI) at the transmitters is not known, but there is knowledge about the signal-to-noise ratio (SNR) of each link. The transmission is carried out over an Additive White Gaussian Noise (AWGN) channel.

We will assume that terminals $T_{1}$ and $T_{2}$ are transmitting Gaussian signals of zero mean and power $P_{1}$ and $P_{2}$, denoted by $x_{1}$ and $x_{2}$. The relay operates in decode-and-forward (DF) mode and it also transmits a Gaussian signal of zero mean and power $P_{\mathrm{RS}}$, denoted by $x_{\mathrm{RS}}$. Two power constraints are considered in the sequel: max-power and sum-average power constraint, both are set to power $P$. In the first case, all the terminals transmit with power $P$, while in the second case the total average power used by the three terminals should be equal to $P$. The details of how $P_{1}, P_{2}$ and $P_{\mathrm{RS}}$ satisfy the power constraints will be given in section IV.

The mutual information of the different links assuming an equal noise power at all terminals is given by,

$$
\begin{aligned}
& I_{X}(P)=N \log _{2}\left(1+\frac{P}{\sigma^{2}}\left|h_{X}\right|^{2}\right), \\
& I_{B C}\left(P_{1}, P_{2}\right)=N \log _{2}\left(1+\frac{P_{1}}{\sigma^{2}}\left|h_{B}\right|^{2}+\frac{P_{2}}{\sigma^{2}}\left|h_{C}\right|^{2}\right)
\end{aligned}
$$

where $X \in\{A, B, C\}$, i.e. $I_{\mathrm{A}}, I_{\mathrm{B}}, I_{\mathrm{C}}$, stand for the mutual information of $\mathrm{T}_{1}-\mathrm{T}_{2}, \mathrm{~T}_{1}$-RS and $\mathrm{T}_{2}$-RS links, and $I_{\mathrm{BC}}$ is the mutual information at the relay when both terminals transmit simultaneously, $\left(\mathrm{T}_{2}, \mathrm{~T}_{1}\right)$-RS link. Finally, $N$ stands for the channel uses employed. The signal models are as follows:

\section{A. Forwarding}

The signal received by the relay according to Figure 1 is,

$$
y_{R S}(k)=\left\{\begin{array}{cc}
h_{B} x_{1}(k)+h_{C} x_{2}(k)+n_{r}(k) & 0 \leq k \leq \alpha_{1} N \\
0 & \alpha_{1} N<k \leq N
\end{array}\right.
$$

where $k$ stands for the channel use employed on each case. The signal received by each terminal is defined by,

$$
\begin{aligned}
& y_{T_{1}}(k)=\left\{\begin{array}{cc}
0 & 0 \leq k \leq \alpha_{1} N \\
h_{B} x_{R S}(k)+n_{1}(k) & \alpha_{1} N<k \leq N
\end{array}\right. \\
& y_{T_{2}}(k)=\left\{\begin{array}{cc}
0 & 0 \leq k \leq \alpha_{1} N \\
h_{C} x_{R S}(k)+n_{2}(k) & \alpha_{1} N<k \leq N
\end{array}\right.
\end{aligned}
$$

where $x_{R S}$ is the signal transmitted by the relay to both terminals. Notice that part of the received signal is seen by each terminal as back-propagating self interference, which can be removed completely if the channel state is known without errors at the receiver.

\section{B. Protocol I}

The signal received by the relay (Figure 2 ) is described by

$$
y_{R S}(k)=\left\{\begin{array}{cc}
h_{B} x_{1}(k)+n_{r}(k) & 0 \leq k \leq \alpha_{1,1} N \\
h_{C} x_{2}(k)+n_{r}(k) & \alpha_{1,1} N<k \leq\left(\alpha_{1,1}+\alpha_{1,2}\right) N \\
0 & \left(\alpha_{1,1}+\alpha_{1,2}\right) N<k \leq N
\end{array}\right.
$$

and the signal received by each terminal is,

$$
\begin{aligned}
& y_{T_{1}}(k)=\left\{\begin{array}{cc}
0 & 0 \leq k \leq \alpha_{1,1} N \\
h_{A} x_{2}(k)+n_{1}(k) & \alpha_{1,1} N<k \leq\left(\alpha_{1,1}+\alpha_{1,2}\right) N \\
h_{B} x_{R S}(k)+n_{1}(k) & \left(\alpha_{1,1}+\alpha_{1,2}\right) N<k \leq N
\end{array}\right. \\
& y_{T_{2}}(k)=\left\{\begin{array}{cc}
h_{A} x_{1}(k)+n_{2}(k) & 0 \leq k \leq \alpha_{1,1} N \\
0 & \alpha_{1,1} N<k \leq\left(\alpha_{1,1}+\alpha_{1,2}\right) N \\
h_{C} x_{R S}(k)+n_{2}(k) & \left(\alpha_{1,1}+\alpha_{1,2}\right) N<k \leq N
\end{array}\right.
\end{aligned}
$$




\section{CODING SCHEME AT THE RELAY}

When the relay is transmitting to both terminals simultaneously the transmission strategy should take into account that terminals are able to remove the backpropagating self interference. The scheme presented in [10][12][13] for the NCR shows that the side information available at the relay is tackled by random binning and the terminals can get rid of the interference.

Another possibility is using XOR precoding, [7][11], which enforces the bit-wise operation at the relay. The relay transmits a single signal devoted to both terminals. This signal uses power $P_{R S}$ and is obtained from the bit-wise operation,

$$
x_{R S}=\hat{x}_{1} \oplus \hat{x}_{2}
$$

where $\hat{x}_{1}$ and $\hat{x}_{2}$ are the estimated signals at the RS. Notice that both messages are inherent in the same signal. To remove the back-propagating self interference each terminal must estimate the signal $x_{R S}$ and apply the bitwise XOR operation of that signal with the previous transmitted one. In such a case, the self interference is cancelled and the signal from the other terminal is obtained. This transmission scheme requires that the transmitted signal by the relay have to be decoded by both terminals. Therefore, the terminal with the worst conditions limits the communication.

\section{RESOURCE ALLOCATION}

The optimal selection of the phase duration, data rate and power allocation are found as the maximization of,

$$
\max _{\boldsymbol{\alpha}, R_{1}, R_{2}, P_{1}, P_{2}, P_{R S}} \mu_{1} R_{1}+\mu_{2} R_{2} \text { s.t. }\left\{\begin{array}{l}
\left(R_{1}, R_{2}\right) \in B(\boldsymbol{\alpha}), 0 \leq f(\boldsymbol{\alpha}) \leq 1 \\
\psi\left(P_{1}, P_{2}, P_{R S}\right) \leq P
\end{array}\right.
$$

where $R_{1}, R_{2}$ stand for the rate transmitted by terminal $\mathrm{T}_{1}$ and $\mathrm{T}_{2}, \alpha$ is a vector that contains the duration of the different phases of the communication, $f$ defines the linear connection between duration of phases, $B(\alpha)$ denotes the achievable rate region for a given $\alpha$ and $\psi$ is a function of the transmitted power, gathering the different power constraints (max and sum-average). Finally, by modifying $\mu_{1}$ and $\mu_{2}$ we attain the boundary of the achievable rate region with optimum phase allocation and rate selection.

We will show through the ensuing subsections that the problem presented in (9) has a unique solution as a function of the protocol, transmission scheme and power constraint employed. Subsections IV.A and IV.B detail the achievable rate region, $B(\alpha)$, and the power constraints $\psi$. Subsection IV.C introduces the resolution.

\section{A. Achievable rate region}

From the signal model presented in (2)-(4), the forwarding protocol presents the following achievable rate region (see [10] for NCR case),

$$
\begin{aligned}
& B^{F W}\left(\alpha_{1}, \alpha_{2}\right)=\left\{\begin{array}{lcl}
R_{1} \leq & \alpha_{1} I_{B}\left(P_{1}\right), & R_{1} \leq \alpha_{2} I_{\Delta}\left(P_{R S}\right) \\
R_{2} \leq & \alpha_{1} I_{C}\left(P_{2}\right), & R_{2} \leq \alpha_{2} I_{\Pi}\left(P_{R S}\right) \\
R_{1}+R_{2} \leq \alpha_{1} I_{B C}\left(P_{1}, P_{2}\right)
\end{array}\right. \\
& f\left(\alpha_{1}, \alpha_{2}\right)=\alpha_{1}+\alpha_{2}
\end{aligned}
$$

The achievable rate region for protocol I, (5)-(7),

$$
\begin{aligned}
& B^{I}\left(\alpha_{1,1}, \alpha_{1,2}, \alpha_{2}\right)=\left\{\begin{array}{l}
R_{1} \leq \alpha_{1,1} I_{B}\left(P_{1}\right) \\
R_{2} \leq \alpha_{1,2} I_{C}\left(P_{2}\right) \\
R_{1} \leq \alpha_{1,1} I_{A}\left(P_{1}\right)+\alpha_{2} I_{\Delta}\left(P_{R S}\right) \\
R_{2} \leq \alpha_{1,2} I_{A}\left(P_{2}\right)+\alpha_{2} I_{\Pi}\left(P_{R S}\right)
\end{array}\right. \\
& f\left(\alpha_{1,1}, \alpha_{1,2}, \alpha_{2}\right)=\alpha_{1,1}+\alpha_{1,2}+\alpha_{2}
\end{aligned}
$$

In both achievable rate regions the values of $I_{\Delta}$ and $I_{\Pi}$ depend on the transmission scheme: NCR or XOR precoding,

$I_{\Delta}=\left\{\begin{array}{ll}I_{C} & \text { if } N C R \\ \min \left(I_{C}, I_{B}\right) & \text { if XOR }\end{array}, I_{\Pi}= \begin{cases}I_{B} & \text { if } N C R \\ \min \left(I_{C}, I_{B}\right) & \text { if XOR }\end{cases}\right.$

\section{B. Power constraints}

Two power constraints are envisioned in this work: terminals transmitting with its maximum power and sum-average power constraint in the three-terminal network. The power transmitted by terminals referenced by $P_{1}, P_{2}, P_{R S}$ in previous sections are transformed in order to satisfy the different power constraints based on a give value of power $P$.

The max-power constraint is typically found in time division access and power limited terminals. In such a case, assuming a maximum power of $P$, the transmitted power of each terminal becomes,

$$
\psi^{\max }\left(P_{1}, P_{2}, P_{R S}\right)=\left\{P_{1}=P, P_{2}=P, P_{R S}=P\right\}
$$

On the other hand, under sum-average power constraint, the transmitted power of each terminal is inversely proportional to its transmission time. Moreover, since the constraint is based on the joint power transmitted by all the terminals, each one is able to use a fraction of the total power, $P$. As a consequence, the instantaneous transmitted power of each terminal is defined by,

$$
\psi^{\text {sum-ave }}=\left\{\begin{array}{l}
P_{1}=\frac{\beta_{1} P}{\alpha_{1}}, P_{2}=\frac{\beta_{2} P}{\alpha_{1}}, P_{R S}=\frac{\beta_{3} P}{\alpha_{2}} \text { if } \mathrm{FW} \\
P_{1}=\frac{\beta_{1} P}{\alpha_{1,1}}, P_{2}=\frac{\beta_{2} P}{\alpha_{1,2}}, P_{R S}=\frac{\beta_{3} P}{\alpha_{2}} \text { if protocol } I
\end{array}\right.
$$

where $\beta_{1}, \beta_{2}$ and $\beta_{3}$ control the fraction of total power $P$ used by each terminal. In order to satisfy the sum-average power constraint $\beta_{1}+\beta_{2}+\beta_{3}=1$.

\section{Solving the resource allocation problem}

The solutions of the problem shown in (9) depend on the power constraint. Under max-power constraint it is possible to obtain closed-form equations for the resource allocation and the sum-rate provided by each protocol. While, under the sumaverage power constraint, we define resource allocation as a convex problem [15], hence there is a unique solution that can be efficiently obtained by interior point methods.

\section{1) Max-power constraint}

When we are interested in maximizing the sum-rate for the forwarding protocol, (10), we must solve,

$$
\alpha I_{B C}-\min \left(\alpha I_{B},(1-\alpha) I_{\Delta}\right)=\min \left(\alpha I_{C},(1-\alpha) I_{\Pi}\right)
$$

The derivations are omitted due to lack of space but are given in [16]. Taking into account the different possibilities the phase duration for forwarding protocol is given by, 


$$
\begin{aligned}
& \int \frac{I_{\Delta}}{I_{B C}+I_{\Delta}-I_{C}} \text { if } \frac{I_{C}}{I_{\Pi}}\left(I_{\Delta}+I_{\Pi}\right) \leq I_{B C}, \frac{I_{B}}{I_{C}} \geq \frac{I_{\Delta}}{I_{\Pi}} \\
& \alpha_{1}^{F W}=\left\{\begin{array}{cl}
\frac{I_{\Delta}+I_{\Pi}}{I_{B C}+I_{\Delta}+I_{\Pi}} & \text { if } \frac{I_{C}}{I_{\Pi}}\left(I_{\Delta}+I_{\Pi}\right)>I_{B C}, \frac{I_{B}}{I_{C}} \geq \frac{I_{\Delta}}{I_{\Pi}} \\
\frac{I_{\Delta}+I_{\Pi}}{I_{B C}+I_{\Delta}+I_{\Pi}} & \text { if } \frac{I_{B}}{I_{\Delta}}\left(I_{\Delta}+I_{\Pi}\right)>I_{B C}, \frac{I_{B}}{I_{C}}<\frac{I_{\Delta}}{I_{\Pi}}
\end{array}\right. \\
& \frac{I_{\Pi}}{I_{B C}+I_{\Pi}-I_{B}} \text { if } \frac{I_{B}}{I_{\Delta}}\left(I_{\Delta}+I_{\Pi}\right) \leq I_{B C}, \frac{I_{B}}{I_{C}}<\frac{I_{\Delta}}{I_{\Pi}} \\
& \alpha_{2}^{F W}=1-\alpha_{1}^{F W}
\end{aligned}
$$

For protocol $I$ we get the following values for the phase duration of phase II from constraints at $R_{1}$ and $R_{2}$ in (11),

$$
\alpha_{2}^{I}=\alpha_{1,2}^{I} \frac{\left(I_{C}-I_{A}\right)}{I_{\Pi}}, \quad \alpha_{2}^{I}=\alpha_{1,1}^{I} \frac{\left(I_{B}-I_{A}\right)}{I_{\Delta}}
$$

After equating them, and considering the time duration constraint, the optimal phase duration is defined by,

$$
\begin{aligned}
& \alpha_{1,1}^{I}=\frac{\left(I_{C}-I_{A}\right) I_{\Delta}}{\left(I_{C}-I_{A}\right) I_{\Delta}+I_{\Pi}\left(I_{B}-I_{A}\right)+\left(I_{B}-I_{A}\right)\left(I_{C}-I_{A}\right)} \\
& \alpha_{1,2}^{I}=\alpha_{1,1}^{I} \frac{\left(I_{B}-I_{A}\right) I_{\Pi}}{\left(I_{C}-I_{A}\right) I_{\Delta}}, \quad \alpha_{2}^{I}=\alpha_{1,2}^{I} \frac{\left(I_{C}-I_{A}\right)}{I_{\Pi}}
\end{aligned}
$$

The sum-rate obtained by each protocol is defined by,

$$
R_{1}+R_{2}= \begin{cases}\alpha_{1}^{F W} I_{B C} & \text { if forwarding } \\ \alpha_{1,1}^{I} I_{B}+\alpha_{1,2}^{I} I_{C} & \text { if protocol } I\end{cases}
$$

\section{2) Sum-average power constraint}

When the sum-average power constraint is assumed for the different protocols and transmission schemes, the resource allocation problem (9) becomes a convex problem for all the variables: data rates, duration of phases in terms of $\alpha_{1}, \alpha_{2}$ for the forwarding and $\alpha_{1,1}, \alpha_{1,2}$ and $\alpha_{2}$ for protocol $I$, and power allocation in terms of $\beta_{1}, \beta_{2}, \beta_{3}$. We can observe that thanks to the power constraint (14), the data rate constraints in (10), (11) depend on $g_{k}$ (or combinations) of two variables,

$$
g_{k}\left(\beta_{k}, \alpha_{k}\right)=\alpha_{k} f\left(\beta_{k} / \alpha_{k}\right), \quad f(x)=\log _{2}(1+x K)
$$

where $\alpha_{k}$ and $\beta_{k}$ stand for a some general variable used in each protocol. From [15] we identify the function $g_{k}$ as the perspective of function $f$. Since this operation preserves the convexity and the function $f$ is concave, so $g_{k}$ is concave. Therefore, all the achievable rate regions, (10), (11), present data constraints based on concave functions. It is important to point out that when the optimization problem (9) is written in standard form [15], it satisfies the common properties of the convex problems.

\section{RESULTS}

The evaluation of the two-way relay-assisted transmission will be carried out in a scenario where both terminals $\left(T_{1}\right.$ and $\left.T_{2}\right)$ are separated by a normalized distance equal to one. The relay is placed at a distance $d$ from terminal $\mathrm{T}_{1}$ and $(1-d)$ from terminal $\mathrm{T}_{2}$. The propagation exponent of each link is equal to 3. The noise power at the different terminals will be the same. We will assume an additive white Gaussian noise channel. Hence the SNR in the different links is given by,

$$
\rho_{A}=\frac{P}{\sigma^{2}} \quad \rho_{B}=\frac{1}{d^{3}} \frac{P}{\sigma^{2}}=\frac{1}{d^{3}} \rho_{A} \quad \rho_{C}=\frac{1}{(1-d)^{3}} \rho_{A}
$$

The OWRC transmission considered in this paper, [14], assumes a four orthogonal phase transmission to account for the transmission of $T_{1}$ to $T_{2}$ and then from $T_{2}$ to $T_{1}$. All the phases are optimized accordingly.

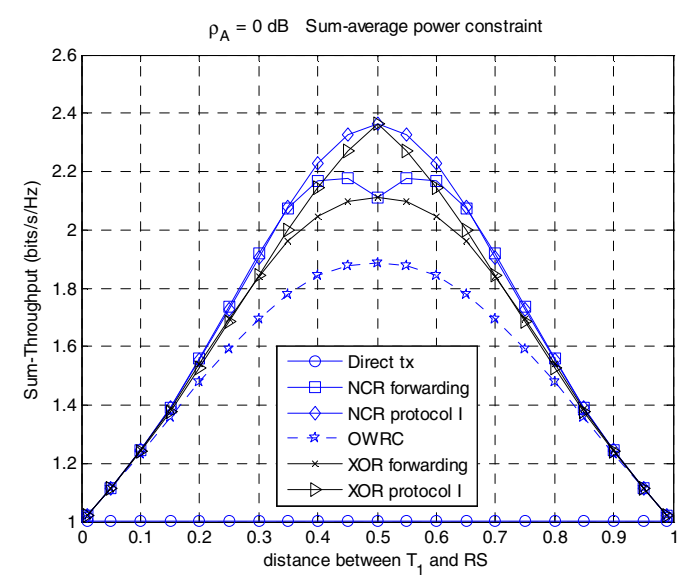

Figure 3. Sum-rate as a function of the position of the RS from $T_{1}$ for the different TWRC and OWRC transmissions when $\rho_{A}=0 \mathrm{~dB}$. Sum-average power constraint

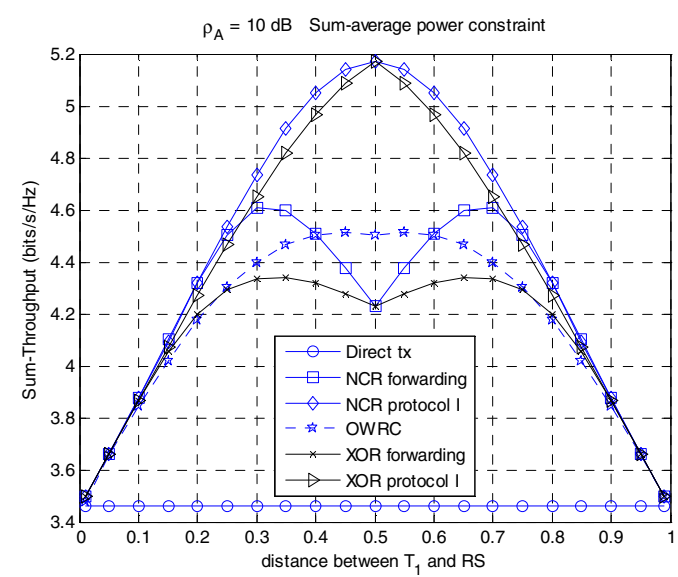

Figure 4. Sum-rate as a function of the position of the RS from $T_{1}$ for the different TWRC and OWRC transmissions when $\rho_{A}=10 \mathrm{~dB}$. Sum-average power constraint

The sum-rate obtained by both terminals according to resource allocation presented in (9) under the sum-average power constraint is investigated in Figure $3\left(\rho_{A}=0 \mathrm{~dB}\right)$ and Figure $4\left(\rho_{A}=10 \mathrm{~dB}\right)$. We observe that in such a case protocol I outperforms both the forwarding protocol and the OWRC transmission. Moreover, XOR precoding with protocol I approaches closely the performance of NCR. In contrast, the forwarding protocol presents higher differences between NCR and XOR. Likewise, the OWRC is able to get better sum-rate than the forwarding protocol at $\rho_{A}=10 \mathrm{~dB}$ in Figure 4.

When we assume a max-power constraint, the sum-rate is given by (19) and the results are shown in Figure $5\left(\rho_{A}=0 \mathrm{~dB}\right)$ and Figure $6\left(\rho_{A}=10 \mathrm{~dB}\right)$. Notice that when those results are compared with the previous figures, many differences come up. Now, the OWRC is able to get better results than protocol $I$ and forwarding for a wide range of positions of the relay 
because $T_{1}$ and $T_{2}$ transmit simultaneously with RS. Hence, those terminals spend more energy in the OWRC than in the TWRC. Moreover, at low SNR, $\rho_{A}=0 \mathrm{~dB}$, the forwarding protocol always gets the best sum-rate (Figure 5), while for middle SNR, $\rho_{A}=10 \mathrm{~dB}$, the position of the relay determines which protocol is better (Figure 6). This performance can be explained by observing the results of the NCR. For the forwarding protocol there are 4 different zones for calculating the sum-rate, (16). The first one describes the situation where the relay moves from $\mathrm{T}_{1}$ up to some point $(d=0.42$ in Figure 5 or $d=0.33$ in Figure 6) while $I_{C}\left(I_{C}+I_{B}\right) \leq I_{B C} I_{B}$. In such a case the sum-rate is equal to $I_{C}$ (using (19),(16) and (12)). The last condition describes the relay approaching to $T_{2}$ and the sumrate becomes $I_{B}$. In those cases the sum-rate obtained by the forwarding protocol is always superior to the one obtained by protocol I, given by (19) with (18) and (12). Nevertheless, for the remaining zones defined in (16), the best protocol depends on the channel configuration as Figure 5 and Figure 6 depict.

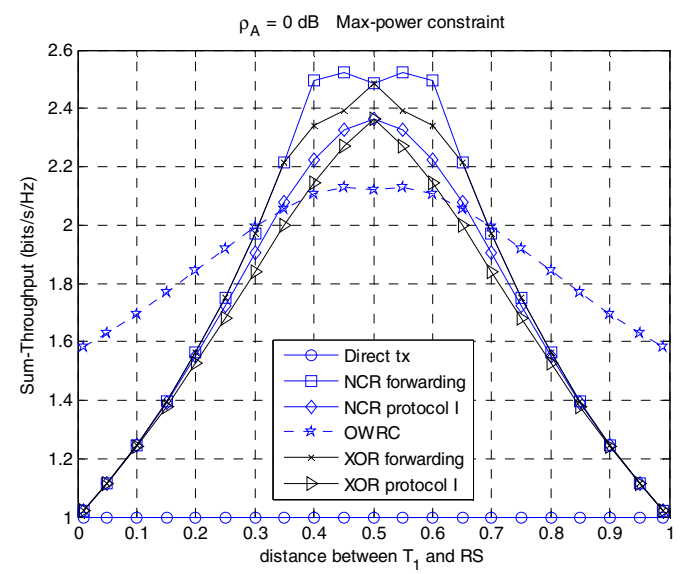

Figure 5. Sum-rate as a function of the position of the RS from $T_{1}$ for the different TWRC and OWRC transmissions when $\rho_{A}=0 \mathrm{~dB}$. Max-power constraint

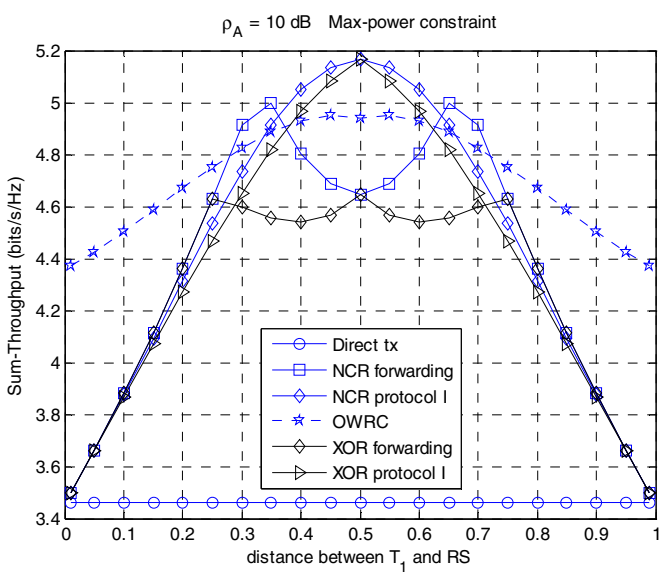

Figure 6. Sum-rate as a function of the position of the RS from $T_{1}$ for the different TWRC and OWRC transmissions when $\rho_{A}=10 \mathrm{~dB}$. Max-power constraint

\section{CONCLUSIONS}

In this paper we have formulated the optimal resource allocation for the TWRC as a convex problem under different power constraints. Two protocols are devised: forwarding and protocol $I$, which have been analyzed in a simple scenario and compared with the best-known OWRC protocol. It has been observed that:

- In terms of efficiency, protocol I is the best one.

- Under a max-power constraint (practical design) the best protocol depends on the position of the relay and the link quality of the channel between terminals $\left(\rho_{A}\right)$. Moreover, OWRC transmission can outperform TWRC transmission.

- Protocol I with XOR precoding is able to get a performance close to the theoretical one obtained with NCR. For the forwarding protocol, there are large differences as a function of the position of the relay.

Further considerations like the introduction of a data rate constraint (i.e. due to the traffic asymmetry in cellular networks) and the use of superposition coding at the relay are deferred to a forthcoming publication, [16].

\section{REFERENCES}

[1] E.C. van der Meulen, "Three-terminal communication channels", Adv. Appl. Prob., vol.3, pp. 120-154, 1971.

[2] T.M.Cover, A.El Gamal, "Capacity theorems for the relay channel", IEEE Trans. Information Theory, vol. 25, no. 5, pp.1468-1489, Sep. 1979

[3] A.Sendonaris, E.Erkip, B.Aazhang, "User cooperation diversity-part I: System description", IEEE Trans. on Communications, vol.51, no.11, pp. 1927-1938, Nov. 2003.

[4] J.N.Laneman, D.N.C.Tse, G.W.Wornell, "Cooperative diversity in wireless networks: Efficient protocols and outage behavior", IEEE Trans. Information Theory, vol. 50, no.12, pp.3062-3080, Dec. 2004.

[5] E.Stauffer, Ö.Oyman, R.Narasimhan, A.Paulraj, "Finite-SNR diversity-multiplexing tradeoffs in fading relay channels", IEEE Journal Selec. Areas in Comm., vol.25, no.2, pp.245-257, Feb. 2007.

[6] A.Agustin, Relay-assisted transmission and Radio Resource Management for Wireless Networks, PhD Thesis, Technical University of Catalonia (UPC), May 2008, Barcelona, Spain

[7] P.Larsson, N.Johansson and K.E. Sunell, "Coded bi-directional relaying”, in Proc. IEEE Vehicular Technology Conf. (VTC-FALL), May 2006, Melbourne, Australia.

[8] B.Rankov, A.Wittneben, "Spectral efficient protocols for half-duplex fading relay channels", IEEE Journal Selec. Areas in Comm., vol. 25, no. 2, Feb. 2007.

[9] B.Rankov, A.Wittneben, "Achievable rate regions for the two-way relay channel", in Proc. IEEE International Symposium on Information Theory (ISIT), July 2006, Seattle, USA.

[10] R.Knopp, "Two-Way Radio Networks with a Star Topology", in IEEE Proc.Intl. Zurich Seminar on Comm. (IZS), Feb.2006, Zurich.

[11] P.Popovski, H.Yomo, "Physical Network Coding in Two-way Wireless Relay Channels", IEEE Proc. of International Conference on Communications (ICC), Jun. 2007.

[12] L.L.Xie, "Network Coding and random binning for multi-user channels", in Proc. IEEE Canadian Workshop on Information Theory (CWIT), Edmonton, Canada, June 2007.

[13] T.J.Oechtering, C.Schnurr, I.Bjelakovic, H.Boche, "Broadcast Capacity Region of Two-phase bidirectional relaying", IEEE Trans. on Information Theory, vol.54, no.1, Jan. 2008.

[14] A. Høst-Madsen, J. Zhang, "Capacity bounds and power allocation for wireless relay channels", IEEE Trans. on Information Theory, vol.51, no.6, pp.2020-2040, June 2005.

[15] S.Boyd, L.Vandenberghe, Convex optimization. Cambridge University Press, 2004.

[16] A.Agustin, J.Vidal, O.Muñoz, "Protocols and optimal resource allocation for the two-way relay channel with half-duplex terminals", submitted to IEEE Trans. on Wireless Comm., Sep. 2008. Temporally available at: http://gps-tsc.upc.es/comm/agustin 